\title{
Study of CP violation in B meson decays in Belle
}

\author{
Y. Yusa*† \\ Niigata University \\ E-mail: yusa@hep.sc.niigata-u.ac.jp
}

We report the measurements of $C P$ violation in $B$ meson decays focusing on the recent studies obtained using a data sample of 772 million $B \bar{B}$ collected by the Belle detector running at the $\Upsilon(4 S)$ resonance at the KEKB $e^{+} e^{-}$collider. We measure the $\phi_{1}$ and $\phi_{2}$, that are angles of the unitary triangle of Cabibbo-Kobayashi-Maskawa (CKM) matrix. Obtained results are consistent with expectations from the Standard Model (SM). Further studies using more statistics in the upgraded $B$-factory experiment are needed for more precise verification and search for the contribution from the new physics.

XIV International Conference on Heavy Quarks and Leptons (HQL2018)

May 27- June 1, 2018

Yamagata Terrsa, Yamagata,Japan

\footnotetext{
* Speaker.

${ }^{\dagger}$ For the Belle Collaboration
} 


\section{Introduction}

In the $\mathrm{SM}, C P$ violation of the quark sector is induced if the complex phase appearing in the CKM matrix which describes the quark mixing [1]. Considering the unitarity between the matrix components in the system containing the $b$-quark, $V_{u d} V_{u b}^{*}+V_{c d} V_{c b}^{*}+V_{u d} V_{u b}^{*}=0$, the $C P$ violation is parameterized as non-zero angles of the unitary triangle, $\phi_{1}=\arg \left[-V_{c d} V_{c b}^{*} / V_{t d} V_{t b}^{*}\right], \phi_{2}=$ $\arg \left[-V_{u d} V_{u b}^{*} / V_{t d} V_{t b}^{*}\right]$ and $\phi_{3}=\arg \left[-V_{c d} V_{c b}^{*} / V_{u d} V_{u b}^{*}\right]$ [2]. Time-dependent $C P$ violation is induced by quantum interference between two decay amplitudes. In the neutral $B$ meson decay, one is box diagram of mixing of $B^{0}$ and $\bar{B}^{0}$, and another is that of the decay into the $C P$-eigenstate. For decays of $B^{0}$ and $\bar{B}^{0}$ mesons produced via $\Upsilon(4 S) \rightarrow B \bar{B}$ transitions, the decay rate has a time dependence $[3,4]$

$$
\mathscr{P}(\Delta t, q)=\frac{e^{-|\Delta t| / \tau_{B^{0}}}}{4 \tau_{B^{0}}} \times\left(1+q\left[\mathscr{S} \sin \left(\Delta m_{d} \Delta t\right)+\mathscr{A} \cos \left(\Delta m_{d} \Delta t\right)\right]\right)
$$

where $\mathscr{S}$ and $\mathscr{A}$ are $C P$-violating parameters; $q=1$ for $B^{0}$ decays and -1 for $\bar{B}^{0}$ decays; $\Delta t$ is the difference in decay times of the $B^{0}$ and $\bar{B}^{0}$ mesons; $\Delta m_{d}$ is the mass difference between the two mass eigenstates of the $B^{0}-\bar{B}^{0}$ system; and $\tau_{B^{0}}$ is the $B^{0}$ lifetime. In this report, we present the results of the $C P$ violation measurement using $772 \times 10^{6} B \bar{B}$ pairs accumulated with the Belle detector [5] on the $\Upsilon(4 S)$ resonance at the asymmetric energy collision of $8.0 \mathrm{GeV} e^{-}$and $3.5 \mathrm{GeV}$ $e^{+}$at the KEKB storage ring [6].

\section{Belle detector}

The Belle detector is a large-solid-angle magnetic spectrometer that consists of a silicon vertex detector (SVD), a 50-layer central drift chamber (CDC), an array of aerogel threshold Cherenkov counters (ACC), a barrel-like arrangement of time-of-flight scintillation counters (TOF), and an electromagnetic calorimeter comprised of $\mathrm{CsI}(\mathrm{Tl})$ crystals (ECL) located inside a super-conducting solenoid coil that provides a $1.5 \mathrm{~T}$ magnetic field. An iron flux-return located outside of the coil is instrumented to detect $K_{L}^{0}$ mesons and to identify muons (KLM). The detector is described in detail elsewhere [5]. Two inner detector configurations were used. A $2.0 \mathrm{~cm}$ radius beampipe and a 3-layer silicon vertex detector was used for the first sample of $152 \times 10^{6} B \bar{B}$ pairs, while a $1.5 \mathrm{~cm}$ radius beampipe, a 4-layer silicon detector and a small-cell inner drift chamber were used to record the remaining $620 \times 10^{6} B \bar{B}$ pairs [7].

\section{Analysis of the $C P$ violation measurement}

In the studies of the $C P$ violation, we reconstruct the decay into the $C P$-eigenstate. Mother $B^{0}$ meson is selected by kinematic variables calculated using the information such as momentum, energy and particle identification from the Belle detector. For the kinematic variables, beam constrained mass $M_{\mathrm{bc}} \equiv \sqrt{\left(E_{\text {beam }} / c^{2}\right)^{2}-\left|\vec{p}_{B}^{\mathrm{CM}} / c\right|^{2}}$ and energy difference $\Delta E \equiv E_{\text {beam }}-E_{B}^{\mathrm{CM}}$ are used, where $\vec{p}_{B}^{\mathrm{CM}}$ and $E_{B}^{\mathrm{CM}}$ are $B$ momentum and energy in the $\Upsilon(4 S)$ center-of-mass system (CMS), respectively. $E_{\text {beam }}$ is a measured beam energy in CMS. To suppress $e^{+} e^{-} \rightarrow q \bar{q}$ continuum background events, a likelihood ratio is calculated using modified Fox-Wolfram moments $[8,9]$ and the 
cosine of the angle between the beam direction and $B^{0}$ flight direction in the CMS, $\cos \theta_{B}$. For the determination of the selection criteria, Monte Carlo simulated events (MC) are used. The signal are generated using the EVTGEN [10] hadronic event generator package. For the background, a large number of $B \bar{B}$ and $q \bar{q}$ processes are simulated. Interactions of the particles in the Belle detector are reproduced using GEANT3 [11] with detector configuration information in each time period of the experiment.

Since the $B^{0}$ flavor $q$ is not specified from observed decay products of the $C P$-eigenstates, $q$ is determined using the information of inclusive properties of particles that are not associated with the signal $B^{0}$ candidate after the selection. For the determination, a multi-dimensional likelihood based method is used[12]. The quality of the flavor tagging result is expressed by $r$, where $r=0$ for no flavor discrimination and $r=1$, where $r=0$ corresponds to no flavor discrimination, and $r=1$ corresponds to unambiguous flavor assignment. Candidates with $r \leq 0.1$ are not used for $C P$ violation measurement. The wrong tag fractions for six $r$ intervals, $w_{l}(l=1-6)$ and their difference between $B^{0}$ and $\bar{B}^{0}$ decays, $\Delta w_{l}$ are determined from a large number of control sample $B^{0}$ decay data in which the flavor is specified by itself. The total effective tagging efficiency defined as $\Sigma\left(f_{l} \times\left(1-2 w_{l}\right)^{2}\right)$ is determined to be $(29.8 \pm 0.4) \%$, where $f_{l}$ is the fraction of the events in the $l$-th interval.

Due to the asymmetric energies of the $e^{+}$and $e^{-}$beams, the $\Upsilon(4 S)$ is produced with a Lorentz boost of $\beta \gamma=0.425$ nearly along the $+z$ axis, which is defined as the direction opposite the $e^{+}$ beam. Since the $B^{0} \bar{B}^{0}$ pair is almost at rest in the $\Upsilon(4 S)$ CMS, the decay time difference $\Delta t$ can be determined from the separation along $z$ of the $B^{0}$ and $\bar{B}^{0}$ decay vertices: $\Delta t \approx \Delta z /(\beta \gamma c)$. The vertex positions of the $C P$ and opposite-side are reconstructed from all charged tracks in the event using a vertex reconstruction algorithm described in [13].

To measure the $C P$ violation parameters, an unbinned maximum likelihood fit is performed for $\Delta t$ and $q$ using the signal fraction evaluated from the signal extraction fit for variables of kinematic and continuum suppression. The PDF for the signal is set to take the form of Eq. 3.1 which is obtained by modifying Eq. 1.1 for wrong-tagging and vertex resolution:

$$
\mathscr{P}(\Delta t, q)=\frac{e^{-|\Delta t| / \tau_{B^{0}}}}{4 \tau_{B^{0}}}\left(1-q \Delta w+(1-2 w) q\left[\mathscr{S} \sin \left(\Delta m_{d} \Delta t\right)+\mathscr{A} \cos \left(\Delta m_{d} \Delta t\right)\right]\right) \otimes R(\Delta t),
$$

where $R(\Delta t)$ is a convolved resolution function which consists of components of detector resolution for $z_{C P}$ and $z_{\text {tag }}$ vertices, the shift of $z_{\text {tag }}$ due to secondary tracks from long-lived particles, and kinematic approximation in the $\Delta t$ calculation from vertex positions. These are determined using a large $C P$-conserving sample of semi-leptonic and hadronic $B$ decays. For the background, which includes both $q \bar{q}$ and $B \bar{B}$, the PDF is modeled as a combination of two Gaussian functions and a $\delta$-function determined using the data sample from the sideband regions of $M_{\mathrm{bc}}$ and $\Delta E$. In the PDF, $\tau_{B^{0}}$ and $\Delta m_{d}$ are fixed to the world average values [14] including modifications to account for the effect of an incorrect flavor assignment. In addition to the signal and background, a broad Gaussian function is included to represent a small outlier component. 


\section{4. $\phi_{1}$ measurement in $B^{0} \rightarrow D^{(*)} h^{0}\left(D \rightarrow K_{S}^{0} \pi^{+} \pi^{-}\right)$decays}

Using the $B^{0}$ decaying into a charmonium meson and $K^{0}$ states, $\sin 2 \phi_{1}$ is measured with good accuracy [15]. A $\sin 2 \phi_{1}$ has two angular solutions for the $\phi_{1}$ so that we need to determine the sign of $\cos \phi_{1}$ for the validation of the SM by comparing $\phi_{1}$ and the other measurements in the unitary triangle. The solution is given by the time-dependent analysis for the $B^{0}$ decays into the multi-body final states. Among the decays, $B^{0} \rightarrow D^{(*)} h^{0}(h=K$ or $\pi)$ followed by $D \rightarrow K_{S}^{0} \pi^{+} \pi^{-}$ has been studied using the full data set of the BABAR and Belle experiments[16]. In this decay, $\cos \phi_{1}$ appears in the coefficient of sine term of the time-dependent decay rate together with the decay amplitude as function of the Dalitz plot variables $M_{K_{S}^{0} \pi^{+}}^{2}$ and $M_{K_{S}^{0} \pi^{-}}^{2}$. Those variables are determined using large number of flavor-specific decay of $D^{*+} \rightarrow D^{0} \pi^{+}$in $e^{+} e^{-} \rightarrow c \bar{c}$ continuum events.

Signal yields of $1129 \pm 48$ and $1567 \pm 56$ events are obtained from the data sample of 471 million $B \bar{B}$ from the BABAR and 772 million $B \bar{B}$ from the Belle, respectively as shown in Figure 1. From the fit to the $\Delta t$ of the selected events, $\sin 2 \phi_{1}=0.80 \pm 0.14$ (stat.) \pm 0.06 (syst.) \pm 0.03 (model) and $\cos 2 \phi_{1}=0.91 \pm 0.22$ (stat.) \pm 0.09 (syst.) \pm 0.07 (model) are obtained. Distribution of propertime intervals and its asymmetries are shown in Figure 2. Significances of the non-zero $\cos \phi_{1}$ and $\sin 2 \phi_{1}$ are $3.7 \sigma$ and $5.1 \sigma$, respectively. Consequently, this is a first evidence of positive $\cos \phi_{1}$ and first observation of the $C P$ violation in $B^{0} \rightarrow D^{(*)} h^{0}$ decays. From this result, a solution of $\phi_{1}=(22.5 \pm 4.4$ (stat.) \pm 1.2 (syst.) \pm 0.6 (model $\left.)\right)^{\circ}$ is favored and this is consistent with other measurements of the unitary triangle.

\section{5. $\sin 2 \phi_{1}^{\text {eff }}$ measurement in $C P$-even states of $B^{0} \rightarrow \pi^{0} \pi^{0} K_{S}^{0}$ decay}

A value of $\sin 2 \phi_{1}$ measured in the decays induced by $b \rightarrow s q \bar{q}(q=u, d, s)$ penguin diagram, $\sin 2 \phi_{1}^{\text {eff }}$, is sensitive to the new physics beyond the SM since the contribution from the new physics is expected to the loop in the diagram. We have measured several kinds of the $C P$-eigenstates [15] and most of the sensitivity is from the $C P$-odd eigenstates of $(q \bar{q}) K_{S}^{0}$. Time-dependent $C P$ violation in the $C P$-even states is measured only in the decays $B^{0} \rightarrow \eta^{\prime} K_{L}^{0}$ and $B^{0} \rightarrow \phi K_{L}^{0}$ since reconstruction of the $B^{0}$ decays including the $K_{L}^{0}$ is difficult. $B^{0} \rightarrow \pi^{0} \pi^{0} K_{S}^{0}$ three-body decay proceeds mainly via a $b \rightarrow s d \bar{d}$ "penguin" amplitude [17] and final state is $C P$-even followed by the $K_{S}^{0}$. We note that there is a $b \rightarrow u \bar{u} s$ tree amplitude that also contributes to $B^{0} \rightarrow K_{S}^{0} \pi^{0} \pi^{0}$ decays and can shift $\phi_{1}^{\text {eff }}$ from $\phi_{1}$; however, this amplitude is doubly Cabibbo-suppressed, and thus the resulting shift is very small [19]. Previously, the BABAR experiment studied this decay and measured $\sin 2 \phi_{1}^{\text {eff }}=-0.72 \pm 0.71 \pm 0.08$ [20]; the sign is opposite to the expectation from the SM although the statistic uncertainty is large.

Since no charged tracks from the decay point of the $B^{0}$ decaying into $C P$ eigenstate, the vertex is determined from the direction of the $K_{S}^{0}$ and constraint from $e^{+} e^{-}$interaction point. Signal yield with the vertex information of $146.7 \pm 23.6$ events are obtained from the three-dimensional fit to variables of kinematic and continuum suppression as shown in Figure 3. Using the selected sample, $\sin 2 \phi_{1}^{\text {eff }}=0.92_{-0.31}^{+0.27}$ (stat.) \pm 0.11 (syst.) and $\mathscr{A}=0.28$ (stat.) \pm 0.04 (syst.) are obtained as shown in Figure 4. This is first result from the Belle experiment and consistent with the SM expectation. 

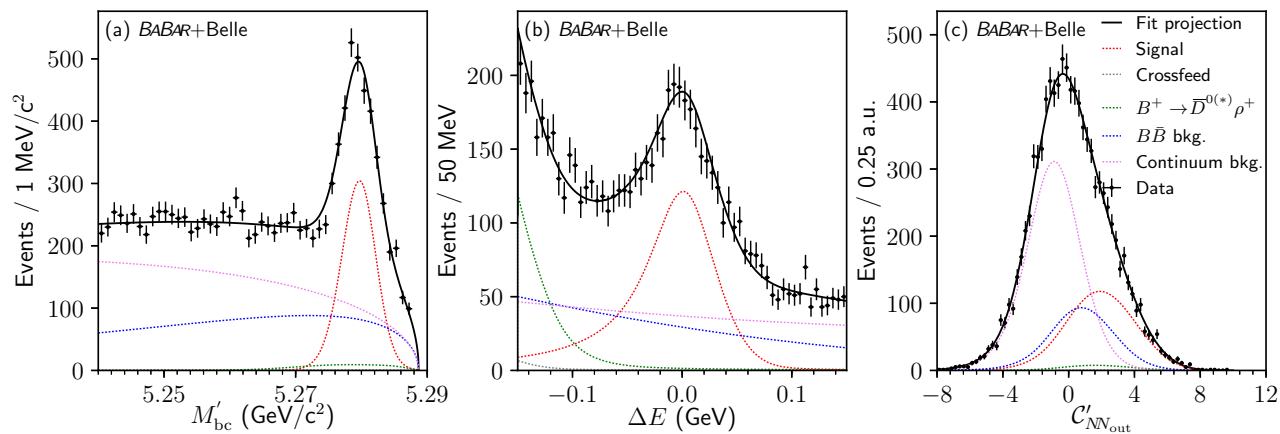

Figure 1: Distributions of $M_{\mathrm{bc}}, \Delta E$ and transformed shape of modified Fox-Wolfram moments $C_{\mathrm{MNN}}^{\prime}$ for selected $B^{0} \rightarrow D^{(*)} h^{0}$ candidates. Points with error bars show the data and solid curve shows the fit result for signal yield extraction. Dotted curves show the contributions from each component of the fit.

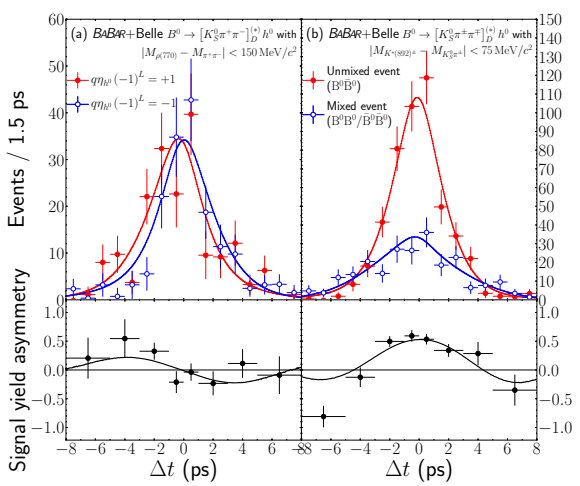

Figure 2: $\Delta t$ and asymmetry distributions of $B^{0} \rightarrow D^{(*)} h^{0}$ candidates. Points with errors show the data together with the fit curves. In the left plot, events are subdivided based on the sign of the sine term in the $\Delta t$ PDF: filled and open circles correspond to positive and negative sign, respectively. In the right plot, filled circle shows the $\Delta t$ of the events in which tagged flavor of the $B$ mesons are different and open circle shows that of the event in which the tagged flavor of the $B$ mesons are same.

\section{Measurement of branching fraction and direct $C P$ violation in $B^{0} \rightarrow \pi^{0} \pi^{0}$ decay and update of $\phi_{2}$ constraint using isospin relation between $B \rightarrow \pi \pi$ system}

As in the case of $\phi_{1}$, the $\phi_{2}$ is measured from the time-dependent decay rate of the decays induced by the $b \rightarrow u \bar{u} d$ tree diagram. On the other hand, measured $\phi_{2}$ contains additional $C P$ phase due to contribution of penguin diagram. Therefore we need to eliminate the additional phase to obtain the $\phi_{2}$. One idea is to use isospin relations between the decay amplitudes of $B \rightarrow \pi \pi$ or $B \rightarrow \rho \rho$ decays [21]. Combining the measurements of branching fractions and $C P$ violations, $\phi_{2}$ is determined with four-fold ambiguity. In the Belle experiment, measurements using 772 million $B \bar{B}$ have been performed for the $B^{0} \rightarrow \pi^{+} \pi^{-}$[22] and $B^{+} \rightarrow \pi^{+} \pi^{0}$ [23].

Branching fraction and direct $C P$ violation in the $B^{0} \rightarrow \pi^{0} \pi^{0}$ are recently updated using full data set. In this decay mode, signal efficiency becomes lower since a photon is lost by the materials installed in front of the ECL in the Belle detector with probability of about $10 \%$ To recover that, remainders detected in the ECL are considered as photon candidates for the $B^{0}$ reconstruction. 

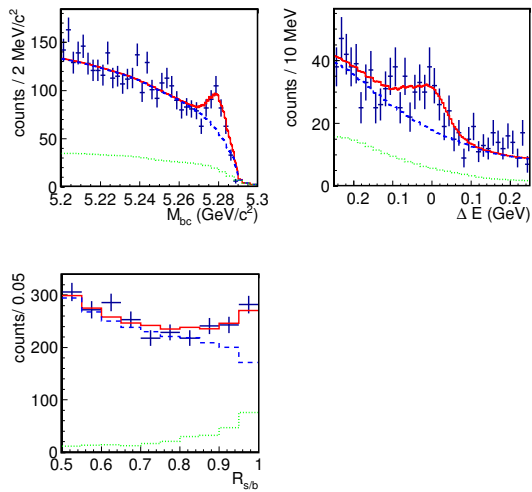

Figure 3: Distributions of $M_{\mathrm{bc}}, \Delta E$ and modified Fox-Wolfram moments $R_{\mathrm{s} / \mathrm{b}}$ for selected $B^{0} \rightarrow$ $\pi^{0} \pi^{0} K_{S}^{0}$ candidates. Points with error bars show the data and solid curve shows the fit result for signal yield extraction. Dashed and dotted curves show the contribution of total and $B \bar{B}$ background, respectively.

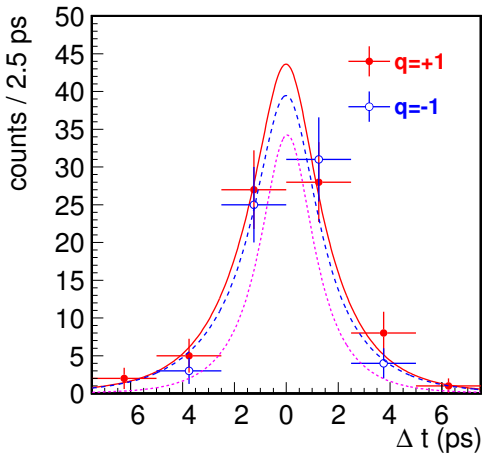

Figure 4: $\Delta t$ distribution of $B^{0} \rightarrow \pi^{0} \pi^{0} K_{S}^{0}$ candidates. Points with errors show the data and events tagged as $q=+1$ and $q=-1$ are shown with filled and open circles together with the fit curves of solid and dashed lines, respectively.

From the extracted signal yields of $217 \pm 32$ event as shown in Figure 5. Branching fraction and direct $C P$ violation parameter are obtained as $(1.31 \pm 0.19$ (stat.) \pm 0.19 (syst. $)) \times 10^{-6}$ and $0.14 \pm$ 0.36 (stat.) \pm 0.10 (syst.), respectively. The significance of the branching fraction is $6.4 \sigma$. By including these result to the isospin relation of $B \rightarrow \pi \pi$ decays, constraint on the $\phi_{2}$ is calculated to $9.5^{\circ}<\phi_{2}<81.6^{\circ}\left(68 \%\right.$ confidence level) and $15.5^{\circ}<\phi_{2}<75.0^{\circ}$ (95\% confidence level) as shown in Figure 6.
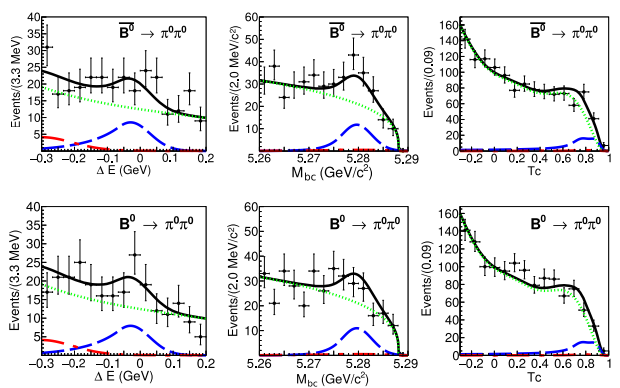

Figure 5: Distributions of $M_{\mathrm{bc}}, \Delta E$ and transformed shape of modified Fox-Wolfram moments $T_{\mathrm{C}}$ for selected $B^{0}\left(\bar{B}^{0}\right) \rightarrow \pi^{0} \pi^{0}$ candidates. Points with error bars show the data and solid curve shows the fit result for signal yield extraction. Dashed, dotted and dot-dashed curves show the contribution of signal, $q \bar{q}$ continuum and $B \bar{B}$ background, respectively.

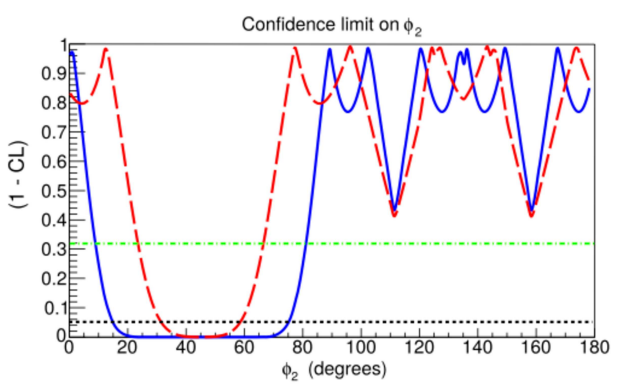

Figure 6: Confidence level vs. $\phi_{2}$ calculated using isospin relation between $B \rightarrow \pi \pi$ decays. This result is based on the measurements in the Belle experiment only. Solid curve shows current bound and dashed curve shows the previous result using $B^{0} \rightarrow \pi^{0} \pi^{0}$ analysis with the data sample of 275 million $B \bar{B}$. Dot-dashed and dotted lines show the confidence levels of $68 \%$ and $95 \%$, respectively. 


\section{Summary}

In summary, we present recent measurements of the $C P$ violation in the decays of $B^{0} \rightarrow D^{*} h^{0}$, $B^{0} \rightarrow \pi^{0} \pi^{0} K_{S}^{0}$ and $B^{0} \rightarrow \pi^{0} \pi^{0}$. Statistical uncertainties are still dominant in most of those analysis and they are expected to be updated using the data from the Belle II [24] experiment in the future.

\section{References}

[1] M. Kobayashi and T. Maskawa, Prog. Theor. Phys. 49, 652 (1973).

[2] Another naming convention, $\alpha\left(=\phi_{2}\right), \beta\left(=\phi_{1}\right)$ and $\gamma\left(=\phi_{3}\right)$ are also used in the literature.

[3] A. B. Carter and A. I. Sanda, Phys. Rev. Lett. 45, 952 (1980); A. B. Carter and A. I. Sanda, Phys. Rev. D 23, 1567 (1981); I. I. Bigi and A. I. Sanda, Nucl. Phys. 193, 85 (1981).

[4] A general review of the formalism is given in I. I. Bigi, V. A. Khoze, N. G. Uraltsev, and A. I. Sanda, “CP Violation” page 175, ed. C. Jarlskog, World Scientific, Singapore (1989).

[5] A. Abashian et al. (Belle Collaboration), Nucl. Instr. and Meth. A 479, 117 (2002); also see detector section in J. Brodzicka et al., Prog. Theor. Exp. Phys. 2012, 04D001 (2012).

[6] S. Kurokawa and E. Kikutani, Nucl. Instr. and. Meth. A 499, 1 (2003), and other papers included in this Volume; T. Abe et al., Prog. Theor. Exp. Phys. 2013, 03A001 (2013) and references therein.

[7] Z. Natkaniec et al. (Belle SVD2 Group), Nucl. Instr. and Meth. A 560, 1(2006).

[8] The Fox-Wolfram moments were introduced in G. C. Fox and S. Wolfram, Phys. Rev. Lett. 41, 1581 (1978). The Fisher discriminant used by Belle, based on modified Fox-Wolfram moments (SFW), is described in K. Abe et al. (Belle Collaboration), Phys. Rev. Lett. 87, 101801 (2001) and K. Abe et al. (Belle Collaboration), Phys. Lett. B 511, 151 (2001).

[9] S. H. Lee et al. (Belle Collaboration), Phys. Rev. Lett. 91, 261801 (2003).

[10] D. J. Lange et al., Nucl. Instr. and Meth. A 462, 152 (2001).

[11] R. Brun et al., CERN DD/EE/84-1 (1984).

[12] H. Kakuno et al., Nucl. Instr. and Meth. A 533516 (2004).

[13] H. Tajima et al., Nucl. Instr. and Meth. A 533370 (2004).

[14] C. Patrignani et al. (Particle Data Group), Chin. Phys. C, 40, 100001 (2016) and 2017 update.

[15] Y. Amhis et al. (Heavy Flavor Averaging Group), Eur. Phys. J. C 77895 (2017).

[16] B. Aubert, et. al. (BABAR Collaboration), arXiv:1804.06152v1 [hep-ex] (2018).

[17] T. Gershon and M. Hazumi, Phys. Lett. B 596163 (2004).

[18] Y. Grossman and M. Woarh, Phys. Lett. B 395241 (1997).

[19] H-. Y. Cheng, arXiv:0702252v1 [hep-ph] (2007).

[20] B. Aubert, et. al. (BABAR Collaboration), Phys. Rev. D 76071101 (2007).

[21] M. Gronau and D. London, Phys. Rev. Lett. 653381 (1990).

[22] J. Dalseno, K. Prothmann, C. Kiesling, et. al. (Belle Collaboration), Phys. Rev. D 88092003 (2013).

[23] Y.-T. Duh, T.-Y. Wu, P. Chang, G. B. Mohanty, Y. Unno, et. al. (Belle collaboration) Phys. Rev. D 87 031103(R) (2013).

[24] T. Abe, et. al. (Belle II Collaaboration), arXiv:1011.0352 [physics.ins-det] (2010). 